\title{
Implicit Brushes for Stylized Line-based Rendering
}

\author{
Romain Vergne $^{1} \quad$ David Vanderhaeghe $^{1,2} \quad$ Jiazhou Chen $^{1,3} \quad$ Pascal Barla $^{1} \quad$ Xavier Granier $^{1} \quad$ Chirstophe Schlick $^{1}$ \\ ${ }^{1}$ INRIA - Bordeaux University $\quad \quad{ }^{2}$ IRIT - Toulouse University $\quad{ }^{3}$ State Key Lab of CAD\&CG - Zhejiang University
}

\begin{abstract}
We introduce a new technique called Implicit Brushes to render animated 3D scenes with stylized lines in realtime with temporal coherence. An Implicit Brush is defined at a given pixel by the convolution of a brush footprint along a feature skeleton; the skeleton itself is obtained by locating surface features in the pixel neighborhood. Features are identified via image-space fitting techniques that not only extract their location, but also their profile, which permits to distinguish between sharp and smooth features. Profile parameters are then mapped to stylistic parameters such as brush orientation, size or opacity to give rise to a wide range of line-based styles.
\end{abstract}

Categories and Subject Descriptors (according to ACM CCS): I.3.3 [Computer Graphics]: Picture/Image Generation-Line and curve generation

\section{Introduction}

Line drawings have always been used for illustration purposes in most scientific and artistic domains. They have also played a fundamental role in the world of animation, mostly because they allow artists to depict the essence of characters and objects with an economy of means. Unfortunately, even when artists restrict drawings to a few clean lines, handdrawn animations require a considerable amount of skills and time. Computer-aided line-based rendering represents an efficient alternative: lines are automatically identified in animated 3D scenes, and drawn in a variety of styles. The challenge is then two-fold: extract a set of salient lines, and render them in a temporally coherent manner.

Most existing line-based rendering techniques consider salient lines as those that best depict the shape of 3D objects. According to the recent study of Cole et al. [CGL*08], there is no consensus among various line definitions. In particular, lines drawn by human subjects do not always represent curvature extrema (ridges or valleys), but may also depict inflections (transitions between convex and concave features). Moreover, lines from different subjects are hardly correlated, as illustrated in Figure 1. It seems that the smoother and less pronounced a surface feature is, the less correlated lines will be, until eventually the feature is too smooth to be depicted by any line at all. The only exception occurs with occluding contours that depict infinitely sharp visibility discontinuities. These observations strongly suggest that on average, lines faithfully represent only these features that exhibit sharpenough profiles. However, a surface feature profile evolves during animation, as the object gets closer or farther from

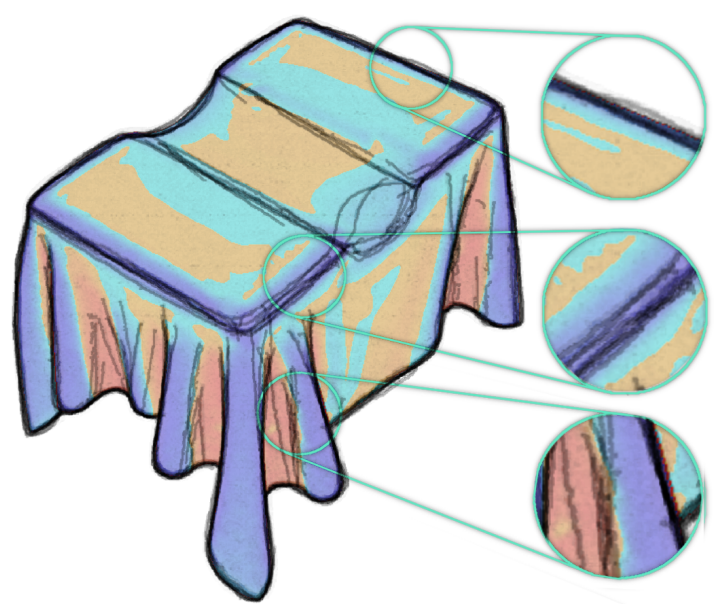

Figure 1: Correlation between hand-drawn lines. Lines drawn by a variety of subjects are accumulated, and either depict concavities (in orange), convexities (in blue) or inflections. Correlation among subjects varies with feature profile sharpness (from top to bottom): lines have high precision at occluding contours, are spread around rounded features, and vaguely located or omitted around smooth features.

the camera, and is rotated or deformed. We thus suggest that lines should be extracted dynamically at each frame, instead of being located onto object surfaces in a preprocess as done in previous work.

The second challenge of line-based rendering is to en- 
sure temporal coherence when lines are stylized. Most previous techniques adopt a stroke-based approach to stylization: they project extracted curves to screen space, parametrize them, and apply a texture along the parametrization to produce a brush stroke. However, surface features, once projected onto the picture plane, are subject to various distortion events during animation: they may either split and merge, or stretch and compress. The stroke-based approach thus raises two important issues: 1) surface features must be tracked accurately and each split or merge event must be handled carefully unless disturbing artifacts due to changes in parametrization will occur; and 2) stroke style must be updated during stretching or compression events unless stylization itself will be stretched or compressed. The simplest alternative for dealing with temporal coherence is to use image-space techniques. However, existing methods are severely restricted both in terms of depicted feature and stylistic choices.

In this paper, we introduce Implicit Brushes, an imagespace line-based rendering technique that permits to depict most salient surface features with a wide variety of styles in a temporally coherent manner. The key idea is to address both challenges of line extraction and temporally coherent stylization with an implicit approach that works in screen space. At each frame, our system identifies surface features located in the vicinity of each pixel, along with feature profile information (Section 3); it then produces a stylized linebased rendering via a convolution process that is only applied to pixels close to features with a sharp-enough profile (Section 4). As a result, stylized lines emerge from the convolution process and dynamically appear or disappear to depict surface features with desired profiles. This approach does not require any temporal feature tracking for handling distortion events, since it relies on the temporal coherence of input data (mainly normal and depth buffers). This is to contrast with stroke-based approaches that introduce temporal artifacts due to parametrization changes.

This approach not only works in real-time with arbitrary dynamic scenes (including deformable objects, even with changing topology), but its performance is independent of 3D scene complexity and it accommodates a number of surface feature definitions, including occluding contours, ridges, valleys and inflections. Thanks to its screen-space definition, it is easily incorporated in compositing pipelines, and works with videos. In terms of stylization abilities, we enrich our convolution-based method with coherent texture techniques inspired by watercolor rendering methods. The result is comparable to the brush tool of raster graphics software such as Photoshop or Gimp. This is to contrast with stroke-based methods, where texture is directly applied to the parametrization, which is similar to the vector graphics styles obtained with software such as Illustrator or Inkscape.

\section{Previous work}

The problem of identifying surface features as curves on $3 \mathrm{D}$ objects has received much attention in previous work. Some authors focus on extracting intrinsic properties of object shape, such as ridges \& valleys (e.g., [OBS04]) or inflections (e.g., Demarcating Curves [KST08]). Although intrinsic surface features are useful to depict purely geometric characteristics, they are not adapted to the goals of stylized rendering where the viewpoint and lighting have an influence on the choice of drawn lines. Undoubtedly, the most important of view-dependent features are occluding contours. They have been extended to Suggestive Contours [DFRS03] (and later Suggestive Highlights [DR07]) to include occluding contours that occur with a minimal change of viewpoint. Apparent Ridges [JDA07] modify ridges \& valleys to take into account foreshortening effects, showing an improved stability in feature extraction compared to Suggestive Contours. Alternative line definitions take light directions into account, as is the case of Photic Extremum Lines [XHT*07] and Laplacian Lines [ZHXC09].

Even if they introduce view- or light-dependent behaviors, all these methods rely on a preprocess that performs differential geometry measurements in object space (except for occluding contours). Surface features are then extracted at runtime from these measurements as zero-crossings in a given direction. The main drawback of this approach is that it completely ignores surface feature profiles after these have been projected to screen-space. As a result, lines depict object features only at a single scale: surface details that would appear in close-up views are ignored, whereas lines appear cluttered when the object is zoomed out. Moreover, deformable objects are not properly depicted since only their rest pose is taken into account for surface measurements. Techniques that precompute measurements at various object-space scales [NJLM06, CPJG09] or for a range of deformations [KNS*09] only partially solve the problem: they do not take projected surface features into account and cannot handle dynamic animations, while requiring user intervention and time- and memory-consuming preprocesses. In contrast, our system extracts new features along with their profile for each new frame, producing line drawings of fully dynamic animations in real-time.

The methods presented so far only produce simple lines. Stroke-based approaches have been introduced to confer varying thickness and textures to extracted lines, by means of a dedicated parametrization. Unfortunately, using a frame-by-frame parametrization leads to severe popping and flickering artifacts; hence static stylization techniques like the system of Grabli et al. [GTDS10] are not adapted to temporally coherent animation. Consequently, specific methods have been devised for the stylization of animated lines. Artistic Silhouettes [NM00] chain occluding contours into long stroke paths, and parametrize them to map a stroke texture. The WYSIWYG NPR system [KMM ${ }^{*}$ 02] uses synthesized stroke textures to ease the creation of novel styles 
that depict occluding contours and creases. Such methods are prone to many temporal artifacts though, which are due to the distortion events mentioned in Section 1. Recent work has tried to address these important issues. The Coherent Stylized Silhouettes of Kalnins et al. [KDMF03] track parametrizations from frame to frame and propose an optimization-based update rule that tries to limit artifacts due to stretching and compression events. The method works in many practical cases, but may also lead to disturbing sliding artifacts. The Self-similar Lines ArtMaps of Bénard et al. [BCGF10] address the same issue by updating texture instead of parametrization, taking advantage of the self-similar nature of many line-based styles.

These stylization techniques deal fairly well with stretching and compression, but they generally fail at dealing properly with splitting and merging, because such changes in line topology necessarily lead to sudden changes in parametrization. Although this may not be too disturbing with occluding contours where lines split or merge mostly around endpoints, other surface features are more problematic. Imagine for instance a pair of parallel ridges that merge together as the object they belong to recedes in the background; in this case, there does not seem to be any natural approach to merge their parametrizations. Our alternative stylization technique avoids the use of parametrizations: it is based on a convolution process that permits a vast range of styles and deals naturally with distortion events.

A simpler solution to line-stylization is to make use of image filters. The pioneering technique of Saito and Takahashi [ST90] explored this idea. Their approach consists in applying simple filters to depth and normal maps in image space to extract occluding contours and creases. It has been adapted to the GPU by Niehaus and Döllner [ND04] using depth peeling for hidden lines and a wobbling effect based on image-space noise for stylization. The method produces coherent line drawings and avoids line-clutter issues by working in screen-space. However, the choice of filter strongly limits both depicted features and line thickness, and the wobbling effect exhibits showerdoor-like artifacts due to the use of a static screen-aligned noise function. To our knowledge, the only filter-based technique that allows lines of controllable thickness is the method of Lee et al. [LMLH07]. It finds edges and ridges in shaded images of 3D objects using a local 2D fitting approach applied to luminance. Although the method is limited to the depiction of luminance features with a simple line style, it shows that a fitting approach in screen-space is able to capture and render dynamic features in real-time. Our approach also makes use of a fitting technique and may thus be seen as a generalization of Lee $e t$ al.'s approach that works with various surface features and provides a richer range of styles.

\section{Feature extraction}

The study of Cole et al. [CGL*08] has shown that although occluding contours are expected to be depicted in virtually all line drawings, other surface features are not systematically drawn. A simple use of occluding contours is not enough though. Regarding this issue, we make no attempt at defining a new kind of surface feature in this paper. Instead, our contribution consists in first providing a screenspace generic definition for most common surface features, then extending it to identify feature profiles (Section 3.1). We then show how these generic surface features are extracted in real-time using an implicit approach that works on the GPU (Section 3.2).

\subsection{Definitions}

In this Section we define common surface features with a generic continuous screen-space formulation. Our choice of domain is motivated by the fact that even for rigid objects, surface features are subject to various distortions if one takes into account their projection in screen-space. For ease of notation, we consider in the following that $\mathbb{R}^{2}$ refers to screenspace.

\subsubsection{Feature Skeleton}

Our first observation is that in most previous methods, features are identified as maxima of a differential geometry invariant in a tangential direction. For instance, a ridge is a local maximum of maximal principal curvature in the maximal principal direction. Similar features are obtained when the analysis is conducted in screen-space. We call the loci of such maxima the feature skeleton. Formally, it is defined by

$$
\mathcal{S}=\left\{\begin{array}{l|l}
\mathbf{s} \in \mathbb{R}^{2} \mid \frac{\delta h(\mathbf{s})}{\delta \theta(\mathbf{s})}=0, \frac{\delta^{2} h(\mathbf{s})}{\delta \theta(\mathbf{s})^{2}}<0
\end{array}\right\},
$$

where $\mathcal{S} \subset \mathbb{R}^{2}, h: \mathbb{R}^{2} \rightarrow \mathbb{R}$ is a $C^{2}$ height function, and $\theta$ : $\mathbb{R}^{2} \rightarrow \mathbb{S}^{2}$ is a $C^{1}$ direction field. Both $h$ and $\theta$ are easily instantiated to produce existing surface feature types.

First-order features are obtained by computing gradients in screen space. Occluding contours are well approximated by taking maxima of the depth gradient $\mathbf{g}_{d}$ in its corresponding direction. Surface Edges are obtained by taking maxima of the surface gradient $\mathbf{g}_{\mathbf{n}}=\left(-n_{x} / n_{z},-n_{y} / n_{z}\right)$ in its corresponding direction, where $\mathbf{n}=\left(n_{x}, n_{y}, n_{z}\right)$ is the surface normal expressed in screen-space. They are very similar to Suggestive Contours computed with the image-space algorithm of DeCarlo et al. [DFRS03], defined as minima of n.v, with $\mathbf{v}$ the view vector. Luminance edges are obtained by taking the maximum of the luminance gradient $\mathbf{g}_{l}$ in its corresponding direction. These features are similar to those of Lee et al. [LMLH07], and may be seen as a screen-space formulation of Photic Extremum Lines [XHT* 07].

Surface ridges and valleys are second-order features, and thus require to compute a curvature tensor $\mathbf{H}$ from which principal curvatures $k_{\max }$ and $k_{\min }$ and directions $\mathbf{t}_{\max }$ and $\mathbf{t}_{\text {min }}$ are extracted. The view-centered tensor of Vergne et al. [VPB*09] provides an appropriate screenspace formulation. Surface ridges (resp. valleys) are obtained as maxima of $k_{\max }\left(\right.$ resp. $-k_{\min }$ ) in the $\mathbf{t}_{\max }$ (resp. 
$\mathbf{t}_{\min }$ ) direction. Contrary to Apparent Ridges [JDA07], $\mathbf{t}_{\max }$ and $\mathbf{t}_{\min }$ are orthogonal in our approach.

Surface inflections are third-order features, and thus require to compute a view-centered curvature-variation tensor $\mathbf{C}$ from $\mathbf{H}$, and extract a curvature gradient $\mathbf{v}_{\max }$ from $\mathbf{C}$. Surface inflections are then obtained as maxima of $\mathbf{v}_{\max }$ in its corresponding direction. They are similar to Demarcating Curves [KST08], but are defined in screen-space.

The set of surface features is summarized in Table 1 .

\begin{tabular}{|l|r|r|}
\hline Names & $h$ & $\theta$ \\
\hline Occluding contours & $\left|\mathbf{g}_{d}\right|$ & $\mathbf{g}_{d} /\left|\mathbf{g}_{d}\right|$ \\
Surface Edges & $\left|\mathbf{g}_{\mathbf{n}}\right|$ & $\mathbf{g}_{\mathbf{n}} /\left|\mathbf{g}_{\mathbf{n}}\right|$ \\
Luminance edges & $\left|\mathbf{g}_{l}\right|$ & $\mathbf{g}_{l} /\left|\mathbf{g}_{l}\right|$ \\
Surface ridges & $k_{\max }$ & $\mathbf{t}_{\max }$ \\
Surface valleys & $-k_{\min }$ & $\mathbf{t}_{\min }$ \\
Surface inflections & $\left|\mathbf{v}_{\max }\right|$ & $\mathbf{v}_{\max } /\left|\mathbf{v}_{\max }\right|$ \\
\hline
\end{tabular}

Table 1: List of common surface features. $\mathrm{g}$ symbols are used for gradients, $k$ for principal curvatures, $\mathbf{t}$ for principal curvature directions and $\mathbf{v}$ for the curvature gradient.

\subsubsection{Feature profile}

An advantage of using Equation 1 is that we can now reason on abstract features without having to focus on a particular definition. As an example, we take the "ripple" function illustrated in Figure 2. It is obvious from this example that the feature skeleton is not sufficient if one wants to convey differences between height field oscillations.

Our second observation is that all the required information to make this distinction is contained in the direction field. Indeed, classic differential geometry [dC76] tells us that for each non-singular point $\mathbf{x}$ of a direction field $\theta$, there exists a unique curve $c_{\mathbf{X}}(t)$ that passes through $\mathbf{x}$ and which tangent is everywhere equal to $\theta$. Such a curve is called a trajectory (or integral curve); a subset of trajectories is drawn as pale blue curves in Figure 2. However, a trajectory may cross multiple times the feature skeleton $\mathcal{S}$. To identify the unique feature profile corresponding to a point $\mathbf{s} \in \mathcal{S}$, we clamp its trajectory to feature mimima (or singularities) on each side of $\mathbf{s}$. This is illustrated in Figure 2 by the dark blue curve. The feature profile $p_{\mathbf{s}}:\left(t_{-}, t_{+}\right) \rightarrow \mathbb{R}$ at a point $\mathbf{s} \in \mathcal{S}$ is defined as the height function along the truncated trajectory:

$$
p_{\mathbf{s}}(t)=h \circ c_{\mathbf{s}}(t), t \in\left(t_{-}, t_{+}\right),
$$

where $t_{+}$(resp. $t_{-}$) is the positive (resp. negative) parametric location of either the closest minimum or nearest singularity.

An interesting property of Equation 2 is that it also applies to any non-singular and non-minimal point $\mathbf{x} \notin \mathcal{S}$. Hence, because of the unicity of a trajectory, for each such point $\mathbf{x}$ lying in the span of a feature profile centered at $\mathbf{s}$ (the dark blue curve in Figure 2), $p_{\mathbf{s}}(t)$ and $p_{\mathbf{x}}(t)$ are equal up to a parametric translation. In other words, a feature skeleton and profile can be obtained implicitly at $\mathbf{x}$ by analyzing a neighborhood along its trajectory. We make use of this property to

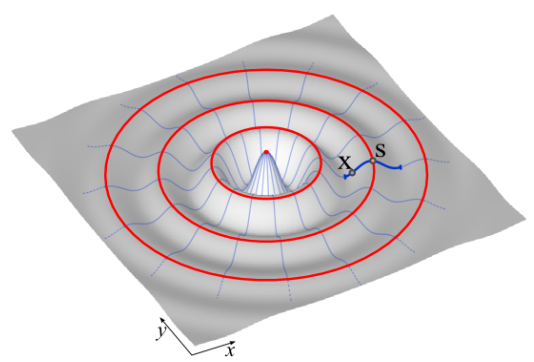

Figure 2: Simple ripple example: we show the height function $h(\mathbf{x})=\cos (|\mathbf{x}|) /(1+0.2|\mathbf{x}|)$, a subset trajectories (in pale blue) of its directional field $\theta(\mathbf{x})=\mathbf{x} /|\mathbf{x}|$, the corresponding feature skeleton $\mathcal{S}$ (in red), and a feature profile (in dark blue) that goes both through points $\mathbf{x} \notin \mathcal{S}$ and $\mathbf{s} \in \mathcal{S}$.

extract feature skeleton and profiles in parallel at each pixel.

\subsection{Implementation}

The extraction of surface features as defined above for a continuous screen-space domain are easily adapted to discrete screen-space. It makes their computation on modern graphics hardware particularly efficient. In our system, this is done in three stages: 1) we compute $h(\mathbf{x})$ and $\theta(\mathbf{x})$ per pixel, using image processing operators; 2 ) we build a $1 \mathrm{D}$ neighborhood for each pixel $\mathbf{x}$ by following its trajectory; 3) we identify feature skeleton and profile along this neighborhood, using a fitting procedure.

\subsubsection{Feature data}

For first-order features, we compute gradients by applying a Sobel filter to the appropriate image buffer: the depth buffer for occluding contours, and the color buffer (averaging color channels) for luminance edges. The surface gradient $\mathbf{g}_{\mathbf{n}}$ required by surface edges is computed from normals, as explained in Section 3.1.1. For second-order features, we compute a view-centered curvature tensor by taking the gradient of $\mathbf{g}_{\mathbf{n}}$ [VPB ${ }^{*}$ 09]. For third-order features, we apply a Sobel filter to mean curvature $H=\operatorname{tr}(\mathbf{H})$ to compute $\mathbf{v}_{\max }$.

Apart for occluding contours that represent pure depth discontinuities, image filters may be applied to screen-space neighborhoods of varying size to capture coarser or finer surface features. However, care must be taken not to make such a neighborhood overlap an occluding contour, otherwise false surface features will emerge. To solve this problem, we follow the approach of Vergne et al. [VPB*09], and apply an iterative anisotropic diffusion to surface or luminance gradients, using occluding contours as insulators. Each iteration corresponds to a filtering pass on the GPU, and the more iterations used, the coarser the features. This process not only smooths $h$ but also $\theta$, resulting in a smaller number of features as shown in the supplemental video.

Having identified $h$ and $\theta$ per pixel for a particular choice of surface feature (see Table 1), we are only one step 


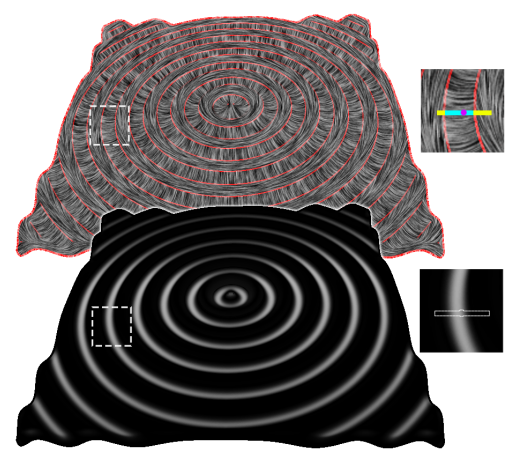

(a)

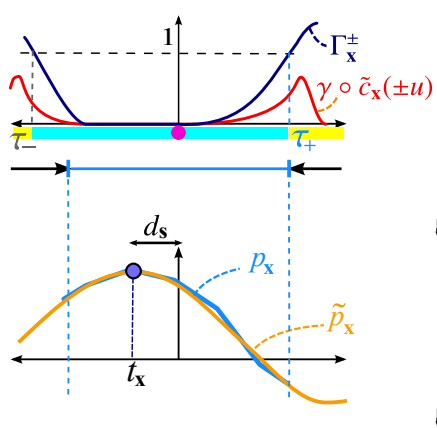

(b)

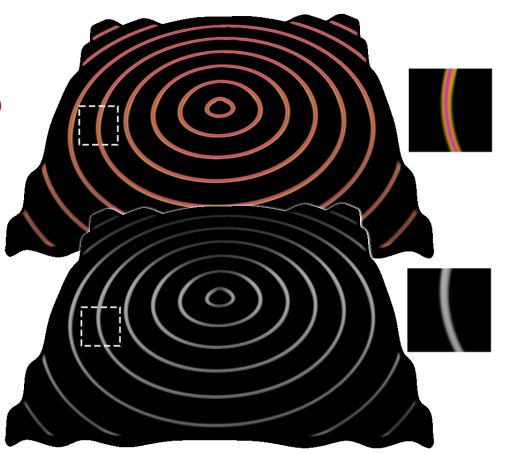

(c)

Figure 3: Feature extraction: (a) Feature data consists of a direction field $\theta$ (here $t_{\text {min }}$, displayed on top using LIC with singularities in red), and a height field $h$ (here $-k_{\min }$, displayed at bottom in gray-scales). (b) The trajectory $\tilde{c}_{\mathbf{x}}(t)$ is shrunk by a factor $\tau_{+}$to stop at feature singularities (top); then profile data $p_{\mathbf{x}}$ is fit using a cubic polynomial $\tilde{p}_{\mathbf{x}}$ (bottom). (c) Profile parameters such as the distance $d_{\mathbf{S}}$ to the skeleton (top) and profile height $\tilde{p}_{\mathbf{x}}\left(t_{\mathbf{x}}\right)$ (bottom) are spatially and temporally coherent.

away from inspecting pixel neighborhoods: we must first locate feature singularities. Singularities of $\theta$ are approximated with the mean angular variation in a 8-pixel neighborhood around each pixel: $\gamma_{\theta}(\mathbf{x})=1-\Sigma_{i=1}^{8}\left|\theta(\mathbf{x}) \cdot \theta_{i}(\mathbf{x})\right| / 8$, with $\theta_{i}(\mathbf{x})$ the orientation at neighboring pixel $i$. Occluding contours must also be considered as singularities, since they delimit non-connex surface neighborhoods. They are approximated by $\gamma_{d}(\mathbf{x})=\left\|\mathbf{g}_{d}(\mathbf{x})\right\|$. Feature singularities are then identified by the union of directional and contour singularities: $\gamma(\mathbf{x})=\max \left(\gamma_{\theta}(\mathbf{x}), \gamma_{d}(\mathbf{x})\right)$. Per-pixel feature data is displayed in Figure 3-a, using Line Integral Convolution [CL93] (LIC) for $\theta$, which is a valid display since all our direction fields are defined modulo $\pi$. We show singularities in red; in this case they appear at places where principal curvatures are of equal magnitude (i.e., at inflection points).

\subsubsection{Profile sampling}

The second stage takes advantage of the observation made at the end of Section 3.1: because each non-generic and nonsingular pixel $\mathbf{x}$ belongs to a unique trajectory $c_{\mathbf{X}}(t)$, we can walk along $c_{\mathbf{x}}(t)$ to find the feature profile it belongs to. In practice, we consider a first-order Taylor expansion of $c_{\mathbf{X}}(t)$ (i.e., a linear neighborhood): $\tilde{c}_{\mathbf{x}}(t)=\mathbf{x}+t \theta(\mathbf{x})$. This approximation is all the more valid for points in the vicinity of $S$ where we have observed that trajectories are close to simple lines. In our system, we measure $p_{\mathbf{x}}(t)$ along $\tilde{c}_{\mathbf{x}}(t)$ at $2 k+1$ samples (henceforth named $t_{i}, i=-k . . k$ ) distributed uniformly on each side of $\mathbf{x}$ (we use $k=4$ ).

However, care must be taken not to go through a feature singularity. To deal with this issue, we take an approach similar to anisotropic diffusion: we shrink $\tilde{c}_{\mathbf{x}}(t)$ as soon as it comes close to a feature singularity. To do that, we first accumulate $\gamma$ values on each side of $\mathbf{x}$ :

$$
\Gamma_{\mathbf{x}}^{ \pm}\left(t_{i}\right)=\sum_{k=0}^{i} \gamma \circ \tilde{c}_{\mathbf{x}}\left(t_{ \pm k}\right)
$$

The neighborhood is then shrunk so that no feature singu- larity is crossed. This is done by identifying the location $\tau_{+}$ (resp. $\left.\tau_{-}\right)$at which $\Gamma_{\mathbf{x}}^{+}\left(\right.$resp. $\left.\Gamma_{\mathbf{x}}^{-}\right)$is greater than a threshold $\Gamma_{\max }$ (we use $\Gamma_{\max }=1$ ), as illustrated at the top of Figure 3(b). The shrinking factor is then taken to be the minimum of $\left|\tau_{-}\right|$and $\left|\tau_{+}\right|$. The shrunk neighborhood is resampled using $2 k+1$ uniformly distributed samples in order to have enough information for profile fitting.

\subsubsection{Profile fitting}

The goal of the third stage of analysis is to identify the location of a potential feature skeleton along the 1D neighborhood, with additional profile information. We do this by fitting an analytic profile function $\tilde{p}_{\mathbf{x}}$ to profile data measured at $t_{i}$ along $\tilde{c}_{\mathbf{x}}(t)$. In practice, we take a least-squares approach, minimizing a per-pixel profile energy on the GPU:

$$
E(\mathbf{x})=\sum_{i=-k}^{k}\left(h \circ \tilde{c}_{\mathbf{x}}\left(t_{i}\right)-\tilde{p}_{\mathbf{x}}\left(t_{i}\right)\right)^{2} .
$$

We use a cubic polynomial for the analytic profile function (see Figure 3-b), since it has just enough degrees of freedom to identify surrounding extrema: $\tilde{p}_{\mathbf{x}}(t)=a t^{3}+b t^{2}+$ $c t+d$. Having a simple analytic expression for the profile at $\mathbf{x}$ allows us to identify characteristic profile properties. The profile generally exhibits two extrema $t_{\alpha, \beta}=$ $\left(-b \pm \sqrt{b^{2}-3 a c}\right) / 3 a$. The skeleton location $t_{\mathbf{x}}$ is easily obtained by picking the one extrema for which the secondorder derivative $d^{2} \tilde{p}_{\mathbf{x}}(t) / d t^{2}=6 a t+2 b$ is positive (when a single minimum is found, we ignore the pixel). Profile height and curvature are then simply given by $\tilde{p}_{\mathbf{x}}\left(t_{\mathbf{x}}\right)$ and $d^{2} \tilde{p}_{\mathbf{x}}\left(t_{\mathbf{x}}\right) / d t^{2}\left(\right.$ since $\left.d \tilde{p}_{\mathbf{x}}\left(t_{\mathbf{x}}\right) / d t=0\right)$.

Figure 3(c) displays results of the fitting process: the perpixel distance to the nearest feature skeleton $d_{\mathbf{s}}=\| \mathbf{x}-$ $\tilde{c}_{\mathbf{X}}\left(t_{\mathbf{x}}\right) \|$ is shown with a color gradient, and profile height $\tilde{p}_{\mathbf{x}}\left(t_{\mathbf{x}}\right)$ is displayed in gray-scales. Observe how both estimates are consistent across a feature profile, illustrating the spatial coherence of per-pixel fitting. Figure 4 compares the 


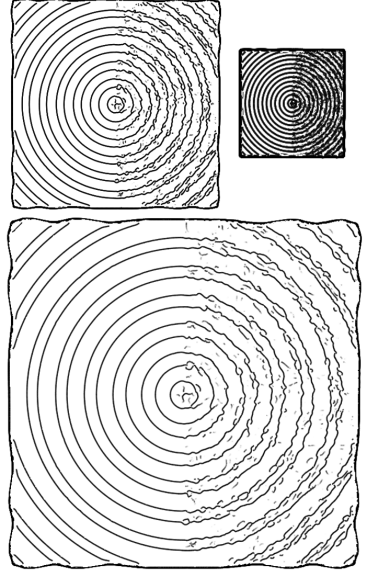

(a)

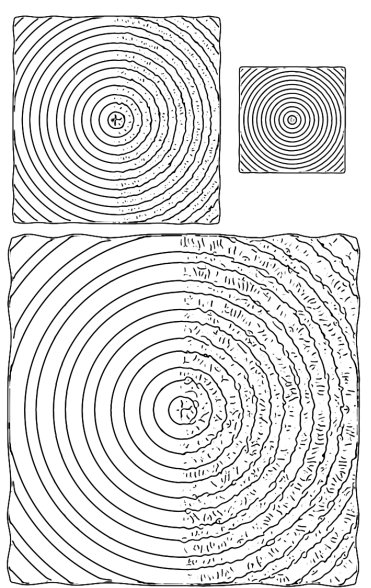

(b)
Figure 4: Comparison with object-space features. (a) Surface features extracted in object-space lead to visual clutter (here Apparent Ridges \& Valleys); (b) our screen-space ridges \& valleys are devoid of this limitation.

feature skeleton extracted with our approach to lines obtained with Apparent Ridges [JDA07] at different scales. Half of the ripple function has been corrupted with noise, which leads to visual clutter in the case of Apparent Ridges. In contrast, our screen-space approach produces clean line drawings, whereby the noise disappears for distant views.

\section{Line stylization}

Depicting dynamic surface features with stylized lines is not straightforward: since features are extracted at each frame, lines need to evolve constantly and coherently to adapt to various changes. Doing so with a stroke-based approach seems cumbersome due to the constantly occurring distortion events we mentioned in Section 1. Our contribution is to provide an alternative stylization technique that is itself defined implicitly. It is based on a convolution approach that mimics the contact of a brush on canvas and easily takes feature profile into account (Section 4.1). We then show how our real-time GPU implementation permits the combination of different brushes and texture effects, and produces a rich variety of styles (Section 4.2).

\subsection{Feature-based convolution}

Intuitively, our stylization technique consists in stamping brush footprints of various sizes, orientations, colors and opacities at points that are close-enough to a feature skeleton with a sharp-enough feature profile. The stylized lines that emerge from this process inherit the spatial and temporal coherence of surface features. It may be seen as an adaptation of Convolution Surfaces [BS91] to the depiction of surface features. As in Section 3, we first present the technique with a generic continuous screen-space formulation.

Formally, an Implicit Brush is a function $\mathcal{I}: \mathbb{R}^{2} \rightarrow$ (a)

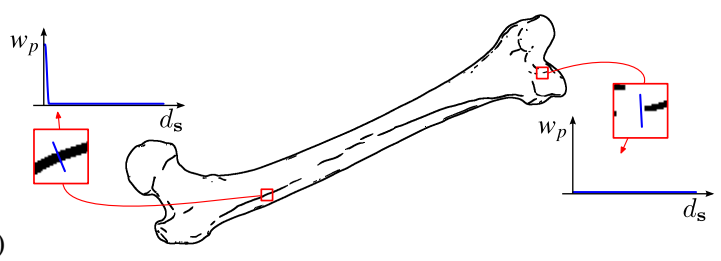

(b)

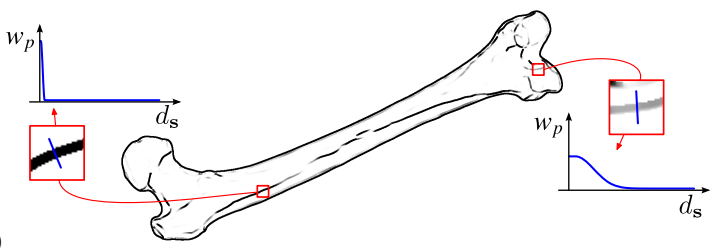

(c)

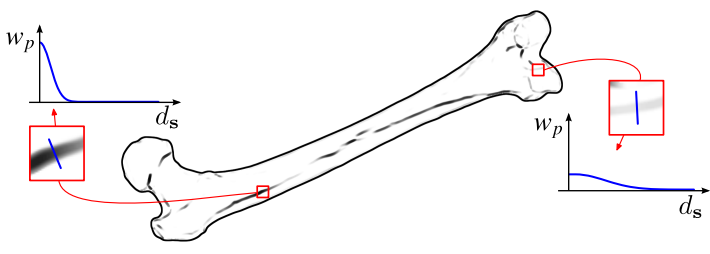

Figure 5: Weight functions. (a) with $\lambda_{p}=1$ and $\sigma_{p}=\varepsilon$, only thin binary lines are selected; $(b)$ with $\lambda_{p}$ proportional to profile height, lines gently disappear with weak features; (c) with $\sigma_{p}$ proportional to profile curvature, lines are smudged around smooth features.

$[0, \infty)^{4}$ that maps a point of the picture plane to a color. It is defined as the convolution of a feature-based weight function $w_{p}: \mathbb{R}^{2} \rightarrow[0,1]$ with a feature-based brush function $b_{p}: \mathbb{R}^{2} \rightarrow[0,1]^{4}$ :

$$
\mathcal{I}(\mathbf{y})=\int_{\mathbb{R}^{2}} w_{p}(\mathbf{x}) b_{p}(\mathbf{y}-\mathbf{x}) d \mathbf{x}
$$

At each point of the image, $\mathcal{I}$ measures the accumulation of weighted footprint contributions for each color channel (including opacity). We remap its values to the $[0,1]^{4}$ range via a homogeneous scaling among color channels as classically done in color tone mapping techniques [Sch94]. The main difference between Implicit Brushes and Convolution Surfaces is that both the weight and brush functions depend on feature profile, as indicated by the $p$ subscript.

The weight function implicitly controls which points of the picture plane are close-enough to a sharp-enough feature. Line-like appearance is ensured by requiring the function to be monotonically decreasing. We use a Gaussian function in our approach:

$$
w_{p}(\mathbf{x})=\lambda_{p} \exp ^{-d_{\mathbf{s}}^{2} / 2 \sigma_{p}^{2}},
$$

where $d_{\mathbf{s}}$ is the distance to the nearest feature skeleton as before, and $\lambda_{p}$ and $\sigma_{p}$ are the feature-based weight peak and standard deviation respectively. Various choices for $\lambda_{p}$ and $\sigma_{p}$ may be made and we show three typical combinations in 
(a)

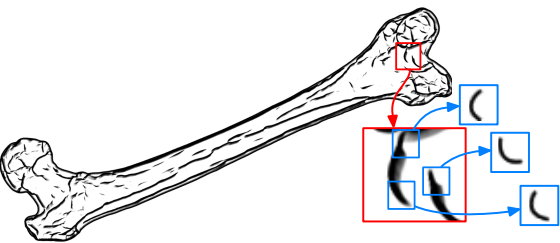

(b)

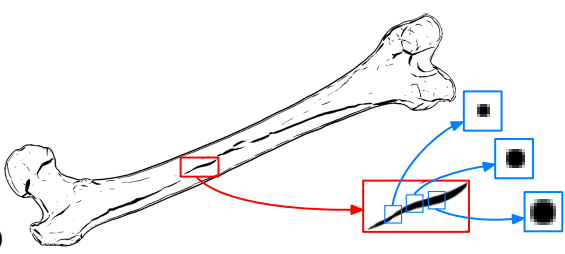

(c)

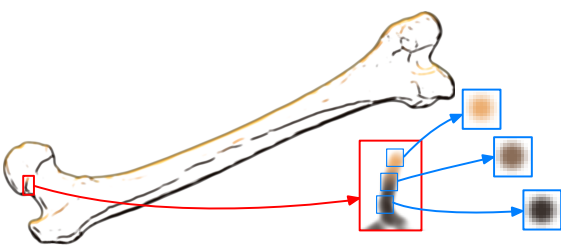

Figure 6: Brush functions. By varying $f_{p}$ and $T_{p}$, we obtain different styles correlated to feature profile properties. (a) $T_{p}$ orient the footprint along the skeleton; (b) $T_{p}$ scale the footprint proportionally to profile height; (c) $f_{p}$ get its color from the shaded $3 D$ object at the skeleton position.

Figure 5, using a small disc footprint. Other combinations are left to user investigations.

The brush function controls how a user-selected brush footprint is applied to local feature data. We separate the footprint from its positioning to allow more user control:

$$
b_{p}(\mathbf{u})=f_{p} \circ T_{p}(\mathbf{u})
$$

where $f_{p}$ is a color footprint function defined in its own parametric space, and $T_{p}$ is a transform that maps a point of the picture plane to this parametric space. We use a similarity transform for $T_{p}$ in our system. Making both functions depend on feature properties permits to correlate style with surface features: $T_{p}$ may orient, scale or move the footprint according to profile properties, while $f_{p}$ may change the color of the footprint, as shown in Figure 6, where we have used the weight function of Figure 5-a. Other combinations of $f_{p}$ and $T_{p}$ are left to user investigations.

Although line style may be strongly correlated to surface features thanks to the choice of weight and brush functions, it is nonetheless independent of the feature extraction process. This is to contrast with previous image-based techniques where extraction and stylization are part of a single process. This is illustrated in Figure 7 where we compare Implicit Brushes with the method of Lee et al. [LMLH07] with identical input data and similar styles. With the method of Lee et al., only opacity can be modulated after the 2D (a)

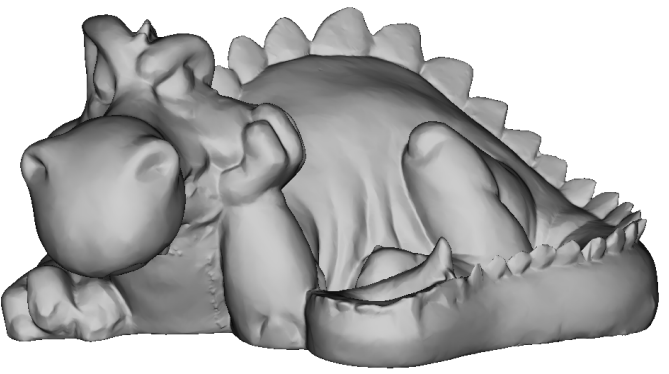

(b)

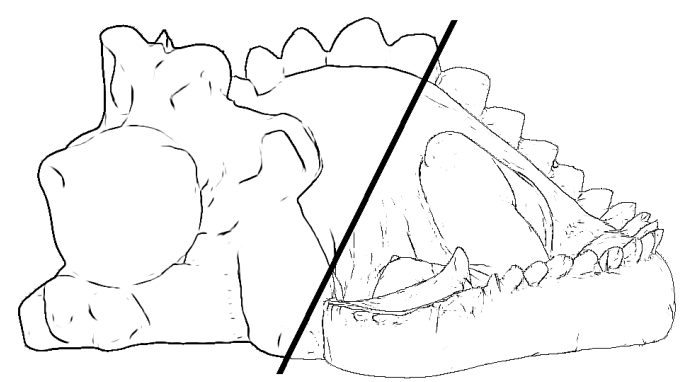

(c)

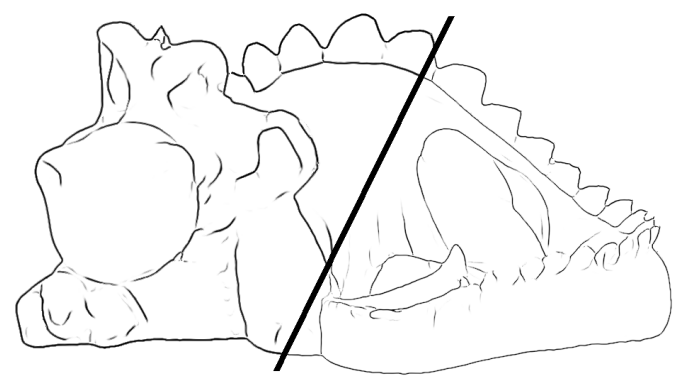

Figure 7: Comparison with Lee et al. [LMLH07]. Rendering luminance valleys from (a) with the method of Lee et al. (b) produces satisfying results for thick-enough lines (left) but raises noise issues with thin lines (right). Opacity thresholds are chosen to obtain the cleanest possible lines. With Implicit Brushes (c), feature extraction and line stylization are controlled independently, which produces clean line renderings for any choice of line thickness.

fitting process, and one must modify feature extraction to control line thickness. As a result, rendering of thin lines requires the identification of small features, which raises noise issues. With our technique, feature extraction is independent of stylization choices, which allows us to create clean renderings even with thin lines.

For aesthetic reasons, it is also necessary to incorporate variations that are not related to any feature property. Examples include the addition of canvas texture, line interruptions, color variation or wobbling effects. In our system, such effects are obtained by modulating each component of Equation 3 with its own noise texture, noted $\eta$ in the following. For instance, the canvas texture effect is obtained by multiplying $\mathcal{I}$ directly by $\eta$; line interruptions are produced by 


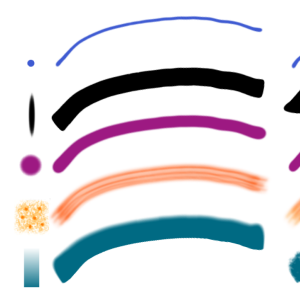

(a)

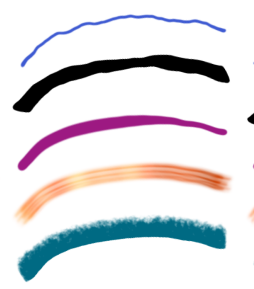

(c)

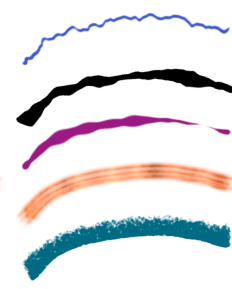

(d)

Figure 8: A few line styles. Each row shows (a) a footprint in its parametric space, $(b)$ applied to a simple curve, (c) with a noise-based small perturbation; or $(d)$ with a more pronounced perturbation. From top to bottom, we perturb brush position, orientation, size, color and add canvas texture.

multiplying $\lambda_{p}$ by $\eta$; color variations are obtained by modulating the color of $f_{p}$; and wobbling effects are created by modulating parameters of $T_{p}$ with $\eta$.

\subsection{Practical applications}

Our prototype system provides a rudimentary brush design interface. A sample of common brush styles is shown in Figure 8: users choose a footprint texture or function (Figure 8a), adjust basic weight and brush parameters (Figure 8-b), and add noise-based perturbation (Figure 8-c-d), observing stylistic variations in real-time. However, as in watercolor rendering techniques, the use of a static screen-aligned noise texture will likely produce sliding artifacts. As of now, there are two known solutions to this issue, both incorporated in our system: textures are either advected off-line using optic flow [BNTS07], or combined at multiple scales to create a fractal texture in real-time [BCGF10].

The main advantage of our convolution-based technique over more complex stylization solutions is that it is fully $d y$ namic: style is entirely controlled by the choice of weight and brush functions, and the algorithm does not require any pre-process, nor does it need to inspect past or future frames, yet ensuring temporal coherence. The method is thus ideally adapted to an implementation on the GPU. Our prototype implementation works in real-time on a NVidia G-480, with a single feature displayed at a time. In practice, we first compute per-pixel weight values and brush transform parameters in a fragment shader, taking extracted feature data as input. An Implicit Brush image is then obtained by rendering one textured quad per pixel with additive blending enabled. Each quad opacity is simply determined by the weight value at its corresponding pixel; it is rotated, scaled and translated according to brush transform parameters; and it is filled using either a bitmap footprint texture or a simple procedural function. The tone mapping operator is applied in a final pass.

Our stylization technique targets applications of various sorts. First, it is well adapted to scientific illustration. Stylized lines are strongly correlated to surface features, as shown in Figure 9 where many small valleys of an engraved stone are depicted with lines where thickness depends on

profile curvature. The method deals equally well with dynamic phenomena like fluids, as seen in the supplemental video. Second, it provides an attractive line-based rendering solution for video games. It works with deformable 3D models such as the animated horse of Figure 10 and naturally depicts features found in normal maps as seen in Figure 11. Third, the method can be used as a building block for the stylization of videos. If normal and depth images are available (e.g., exported from a 3D rendering application), then multiple feature types may be depicted in a single image by applying our technique on each type of feature and compositing them. This is illustrated in Figure 12, which shows a fly sequence over a terrain model with ridges, valleys and occluding contours rendered in different ways. Our method may also be applied to a standard video, as seen in the water drop sequence of Figure 13 where stylized lines are either drawn alone or overlayed on top of original images. In this case, we use luminance edges and make only use of directional singularities since occluding contours are unavailable.

\section{Discussion and future work}

We have presented two implicit techniques for surface feature extraction and line stylization that, when used in combination, permit to create coherent stylized line-based renderings of dynamic 3D scenes in real-time. We avoid visual clutter thanks to a screen-space definition that extracts generic features which profile is relevant at a userchosen scale. Our stylization technique produces lines that are strongly correlated to depicted feature profiles. It is also naturally coherent, without requiring preprocessing or tracking: this is because features are extracted from temporally coherent normal and depth buffers, and we preserve such coherence through fitting and convolution.

The performance of our system is mainly dependent on fill-rate: it varies with image resolution, the number of anisotropic diffusion iterations, and footprint size. For example, using a NVidia G-480, it runs at $82 \mathrm{fps}$ at a resolution of $1024 \times 768$ with a footprint of radius 10 pixels. Performances drop down with increasing iterations: it runs at 56, 40 and 31 fps for 10, 20 and 30 iterations respectively.

Our feature extraction technique works for a range of surface feature types, including edges, ridges, valleys, occluding contours and inflections. However, the choice of feature has an influence on a feature profile extent: indeed, with feature definitions of increasing order, more singularities occur, which leads to more profile clamping on average. Another limitation of our approach is that it ignores junctions, precisely because they are located at directional singularities. An exciting direction of future work would thus be to find a novel feature definition that limits the number of singular points, while easing the identification of junctions. Our surface features often exhibit natural behaviors when objects recede in the background. This is due to our screen-space algorithm that implicitly merges features based on their distance in the picture plane. The quality of rendered lines for distant 

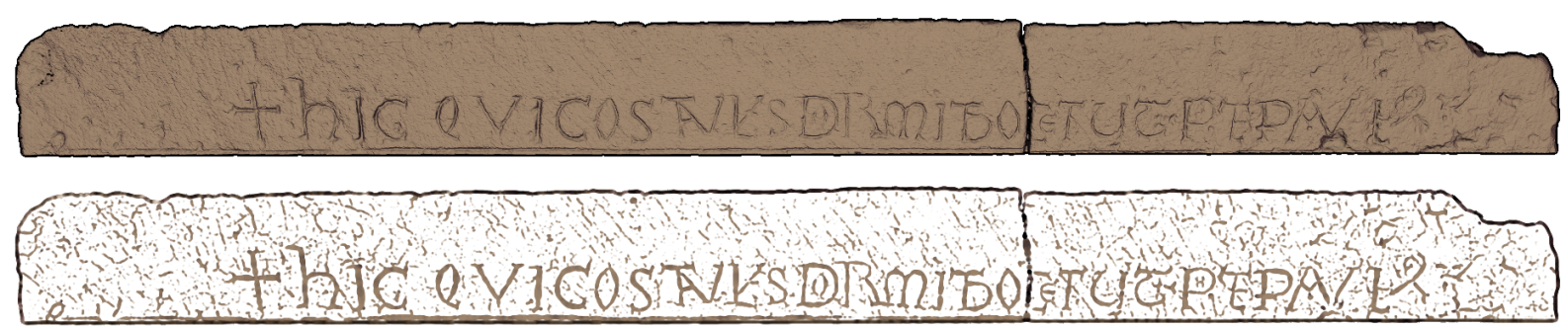

Figure 9: Rendering a carved stone. Both engraved symbols and details of this stone correspond to surface valleys, although with different profiles. We convey this distinction by varying disk footprint size according to profile curvature.
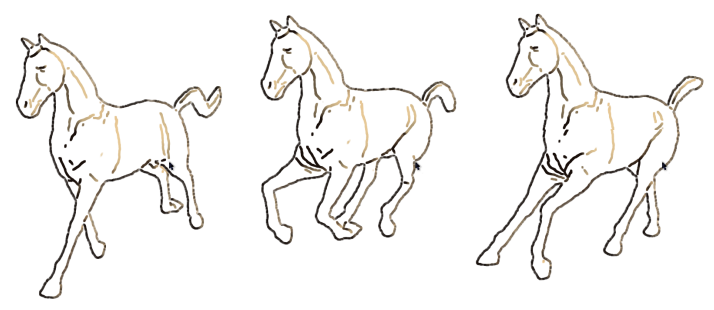

Figure 10: A deformable 3D model. Frames of an animated $3 D$ horse model rendered with shaded surface valleys.

objects is sensitive to aliasing though: flickering artifacts occur if no anti-aliasing technique is used. Moreover, such a systematic simplification may not always be adapted. For familiar objects, one may want to preserve important features (e.g., around the eyes) in multiple views; for structured objects such as grid- or wave-like patterns, simplification may not be robust to small viewpoint variations. We would like to investigate object-space semantic simplification techniques in future work to complement our approach.

Our stylization technique produces temporally coherent lines from dynamic 3D scenes in a variety of styles. In comparison, other methods are either limited in terms of stylization abilities [ND04, LMLH07], since they only provide thickness control; or they are prone to temporal artifacts even on static objects [KDMF03, BCGF10], mainly with split or merge events. The flexibility of our approach comes at a price though: user control is less direct with Implicit Brushes than with stroke-based techniques. For instance, perturbations are produced via a global screen-space texture; and stylized lines automatically end at feature end-points based on the choice of weight function. In other words, there is no simple solution for applying a stroke texture along a line. Although it is a limitation that prevents accurate local control of line style, it may also be seen as an advantage for the applications we target in this paper: style is designed once and for all and applied either in real-time for dynamic systems, or as a batch process for compositing pipelines. However, a more direct and local control in both space and time might be required, for special effects applications for instance. In such cases, our stylization technique is not adapted, but our feature extraction technique is still relevant. A promising av-

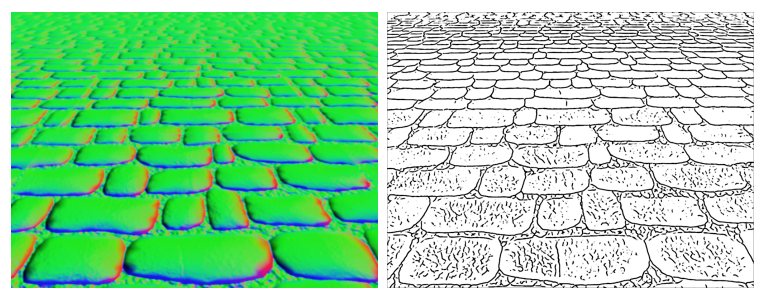

Figure 11: Rendering normal maps. Since our method works from normal buffers, it deals naturally with normalmapped objects, here with surface valleys.

enue of future work would be to extend the WYSIWYG approach $\left[\mathrm{KMM}^{*} 02\right]$ to depict dynamic surface features.

\section{Acknowledgments}

We would like to thank Forrester Cole for providing handdrawn line data used in Figure 1, and the Aim@Shape library for 3D models of Figures 5, 6 and 10. This work has been sponsored by the Animare (ANR-08-JCJC-0078-01) and SeARCH (ANR-09-CORD-019) projects, and the INRIA postdoctoral program.

\section{References}

[BCGF10] Bénard P., Cole F., Golovinskiy A., FinkelSTEIN A.: Self-similar texture for coherent line stylization. In Proc. NPAR (2010), ACM, pp. 91-97.

[BNTS07] Bousseau A., Neyret F., Thollot J., Salesin D.: Video watercolorization using bidirectional texture advection. ACM TOG (Proc. SIGGRAPH) 26, 3 (2007).

[BS91] Bloomenthal J., Shoemake K.: Convolution surfaces. In Proc. SIGGRAPH (1991), vol. 25, ACM, pp. 251-256.

[CGL*08] Cole F., Golovinskiy A., Limpaecher A., Barros H. S., Finkelstein A., Funkhouser T., Rusinkiewicz S.: Where Do People Draw Lines? ACM TOG (Proc. SIGGRAPH 2008) 27, 3 (2008), 1-11.

[CL93] CABRAL B., LEEDOM L. C.: Imaging vector fields using line integral convolution. In Proc. SIGGRAPH (1993), ACM, pp. 263-270.

[CPJG09] Cipriano G., Phillips JR. G. N., Gleicher M.: Multi-scale surface descriptors. IEEE TVCG 15, 6 (2009), 12011208.

[dC76] Do CARMo M. P.: Differential Geometry of Curves and Surfaces. Prentice-Hall, Englewood Cliffs, NJ, 1976. 

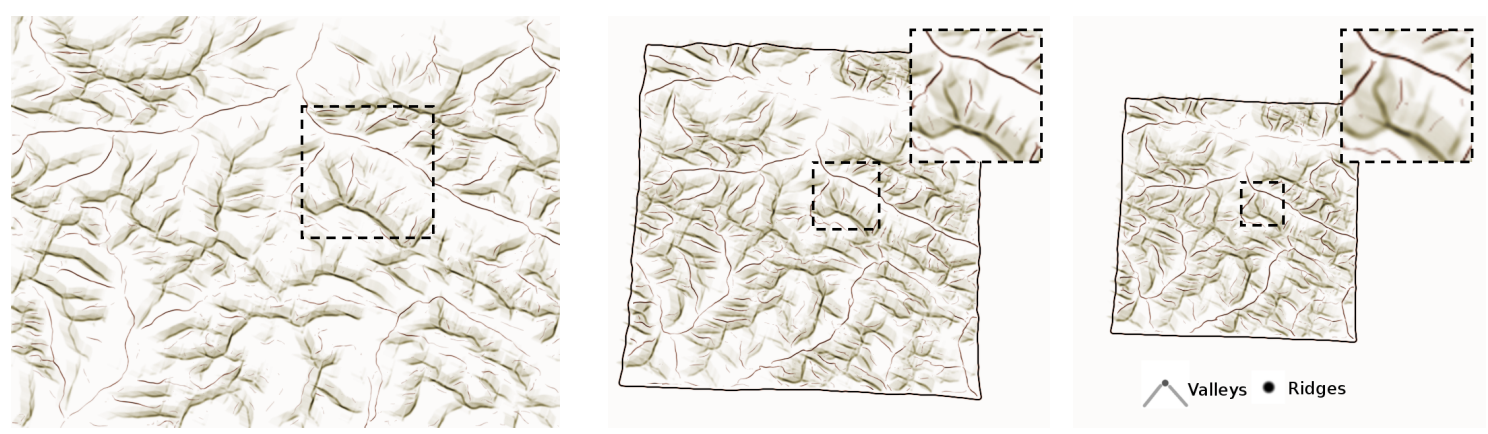

Figure 12: Composited features. A 3D terrain model rendered with two different styles for ridges and valleys. Surface features are simplified automatically at different scales, and the corresponding stylized lines are merged coherently.
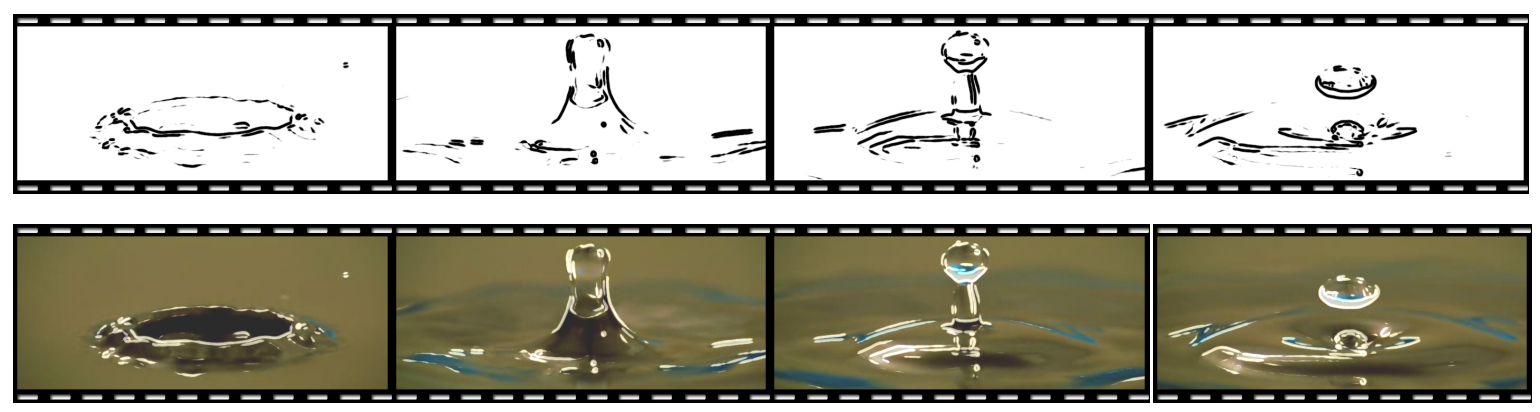

Figure 13: A stylized video. Frames of a water drop video where luminance edges are depicted with lines of varying thickness, either drawn in black (top), or drawn in white over the original image (bottom), both with a disk footprint.

[DFRS03] DeCarlo D., Finkelstein A., Rusinkiewicz S., SANTELla A.: Suggestive Contours for Conveying Shape. ACM TOG (Proc. SIGGRAPH) 22, 3 (2003), 848-855.

[DR07] DeCARLO D., RUSinkiewicz S.: Highlight lines for conveying shape. In Proc. NPAR (2007), ACM, pp. 63-70.

[GTDS10] Grabli S., Turquin E., Durand F., Sillion F. X.: Programmable rendering of line drawing from $3 \mathrm{~d}$ scenes. ACM TOG 29, 2 (2010), 1-20.

[JDA07] Judd T., DuRAND F., Adelson E. H.: Apparent Ridges for Line Drawing. ACM TOG (Proc. SIGGRAPH) 26, 3 (2007), 19.

[KDMF03] Kalnins R. D., Davidson P. L., Markosian L., FINKELSTEIN A.: Coherent stylized silhouettes. ACM TOG (Proc. SIGGRAPH) 22, 3 (2003), 856-861.

[KMM*02] Kalnins R. D., Markosian L., Meier B. J., Kowalski M. A., Lee J. C., Davidson P. L., WebB M., HugHES J. F., FinKELSTEIN A.: Wysiwyg npr: drawing strokes directly on 3d models. ACM TOG (Proc. SIGGRAPH) 21, 3 (2002), 755-762.

[KNS*09] Kalogerakis E., Nowrouzezahrai D., Simari P., McCrae J., Hertzmann A., Singh K.: Data-driven curvature for real-time line drawing of dynamic scene. ACM TOG 28, 1 (2009).

[KST08] Kolomenkin M., Shimshoni I., TAL A.: Demarcating Curves for Shape Illustration. ACM TOG (Proc. SIGGRAPH Asia) 27, 5 (2008), 1-9.

[LMLH07] Lee Y., Markosian L., Lee S., Hughes J. F.: Line drawings via abstracted shading. ACM TOG (Proc. SIGGRAPH) 26, 3 (2007), 18.
[ND04] Nienhaus M., Döllner J.: Blueprints: illustrating architecture and technical parts using hardware-accelerated nonphotorealistic rendering. In Proc. GI (2004), pp. 49-56.

[NJLM06] Ni A., Jeong K., Lee S., Markosian L.: Multiscale line drawings from $3 \mathrm{~d}$ meshes. In Proc. I3D (New York, NY, USA, 2006), ACM, pp. 133-137.

[NM00] NORTHRUP J. D., MARKosian L.: Artistic silhouettes: a hybrid approach. In Proc. NPAR (2000), ACM, pp. 31-37.

[OBS04] OhtaKe Y., Belyaev A., SeIDEl H.-P.: Ridge-valley lines on meshes via implicit surface fitting. ACM TOG (Proc. SIGGRAPH) 3, 23 (2004), 609-612.

[Sch94] SCHLICK C.: Quantization techniques for visualization of high dynamic range pictures. In Proc. EGSR (1994), SpringerVerlag, pp. 7-20.

[ST90] SaITo T., TAKahashi T.: Comprehensible rendering of 3-d shapes. vol. 24, ACM, pp. 197-206.

[VPB*09] Vergne R., PaCanowski R., Barla P., Granier X., SCHLICK C.: Light warping for enhanced surface depiction. ACM TOG (Proc. SIGGRAPH) 28, 3 (2009).

[XHT*07] Xie X., He Y., Tian F., Seah H.-S., Gu X., Qin H.: An effective illustrative visualization framework based on photic extremum lines. IEEE TVCG 13, 6 (2007), 1328-1335.

[ZHXC09] Zhang L., He Y., XIE X., Chen W.: Laplacian Lines for Real Time Shape Illustration. In Proc. I3D (2009), ACM. 